\title{
The Effectiveness of Ruxolitinib for Acute/ Chronic Graft-versus-Host Disease in Children: A Retrospective Study
}

This article was published in the following Dove Press journal: Drug Design, Development and Therapy

\author{
Wenli Yang, ', Guanghua Zhu, ${ }^{2, *}$ \\ Maoquan Qin, ${ }^{2}$ Zhigang $\mathrm{Li}^{3}$ \\ Bin Wang, Jun Yang, ${ }^{2}$ \\ Tianyou Wang (iD ${ }^{2}$ \\ 'Department of Clinical Nutrition, Beijing \\ Children's Hospital, Capital Medical \\ University, National Center for \\ Children's Health, Beijing, People's \\ Republic of China; ${ }^{2}$ Department of \\ Hematology and Oncology, Beijing \\ Children's Hospital, Capital Medical \\ University, National Center for \\ Children's Health, Beijing Key Laboratory \\ of Pediatric Hematology Oncology, Key \\ Laboratory of Major Diseases in \\ Children, Ministry of Education, National \\ Key Discipline of Pediatrics, Beijing, \\ People's Republic of China; ${ }^{3}$ Hematology \\ and Oncology Laboratory, Beijing \\ Pediatric Research Institute, Beijing \\ Children's Hospital, Capital Medical \\ University, National Center for \\ Children's Health, Beijing Key Laboratory \\ of Pediatric Hematology Oncology, Key \\ Laboratory of Major Diseases in \\ Children, Ministry of Education, National \\ Key Discipline of Pediatrics, Beijing, \\ People's Republic of China
}

*These authors contributed equally to this work

Correspondence: Tianyou Wang Department of Hematology and Oncology, Beijing Children's Hospital, Capital Medical University, National Center for Children's Health, Beijing Key Laboratory of Pediatric Hematology Oncology, Key Laboratory of Major Diseases in Children, Ministry of Education, National Key Discipline of Pediatrics, Nanlishi Road No. 56, Xicheng District, Beijing 100045, People's Republic of China

Tel +86 I0 596I 7I23

Fax +861059718700

Email wangtiany_wty@।63.com
Objective: This study aimed to evaluate the effectiveness of Ruxolitinib for acute/chronic graft-versus-host disease in children.

Methods: This study was a retrospective trial. We analyzed the clinical characteristics of children who responded poorly to previous treatment for graft-versus-host disease (GVHD) and received ruxolitinib treatment after allogeneic hematopoietic stem cell transplantation (allo-HSCT) as an additional or replacement therapy.

Results: A total of 53 patients were analyzed: aGVHD and cGVHD. The overall response rate (ORR) to ruxolitinib was $75.5 \%$. The ORR was $64.7 \%(11 / 17)$ in the aGVHD group including 6, 5, and 6 patients with partial responses (PRs), complete responses (CRs), and treatment failure, respectively. The ORR was $80.6 \%$ (29/36) in the cGVHD group including 10 with CRs and 19 with PRs. Five and 2 patients showed no response and treatment failure, respectively. Four and 14 patients were GVHD recurrence in aGVHD and cGVHD respectively. A total of 14 patients (39\%) discontinued steroids and 8 patients $(22.2 \%)$ reduced steroids. The incidence of obvious adverse events was $94.1 \%(16 / 17)$ in the aGVHD group, which was higher than that in the cGVHD group. Meanwhile, the prognosis of children with cGVHD was superior to that of children with aGVHD after treatment with ruxolitinib. During the ruxolitinib treatment, only 1 patient suffered a relapse of the primary tumor. Eleven patients also suffered transplantation-associated thrombotic microangiopathy (TATMA) after allo-HSCT.

Conclusion: Pediatric patients with GVHD (especially cGVHD) responded well to ruxolitinib treatment. Ruxolitinib can also be used as an alternative treatment for patients with TMA.

Keywords: ruxolitinib, graft-versus-host disease, allogeneic hematopoietic stem cell transplantation, Janus kinase $1 / 2$ inhibitor, pediatric patients

\section{Introduction}

Allogeneic hematopoietic stem cell transplantation (allo-HSCT) is an important treatment for malignant hematological diseases, hereditary diseases, and some solid tumors. However, graft-versus-host disease (GVHD) is common after allo-HSCT. $40-60 \%$ of patients develop grade II-IV acute GVHD (aGVHD) and the incidence of chronic GVHD (cGVHD) is as high as $80 \%$ and the overall case-fatality rate can reach $35 \%$ or even higher. ${ }^{1}$ Thus, GVHD remains a major complication and the leading cause of death among allo-HSCT recipients. ${ }^{1}$ Methylprednisolone $1-2 \mathrm{mg} / \mathrm{kg} /$ day or equivalent is the first-line therapy for acute grades II-IV GVHD and the 
response rate to hormone monotherapy is $50 \% .^{2}$ There is currently no standard treatment for patients with steroidrefractory (SR)-GVHD. Second-line treatments including mycophenolate mofetil (MMF), tacrolimus, rapamycin, monoclonal antibodies, and extracorporeal photochemotherapy (often used in combination) have been applied during treatment efforts, although, with poor efficacy. ${ }^{1}$

In recent years, ruxolitinib, a selective nonreceptor Janus kinase (JAK) 1/2 inhibitor, has been used to treat a/cGVHD. Both animal experiments and clinical studies in adults have demonstrated that ruxolitinib leads to a partial response $(\mathrm{PR})$ in experimental animals or patients with SR-GVHD. ${ }^{3-6}$ What's more, ruxolitinib has recently been FDA-approved for SR-GVHD. ${ }^{7}$ Although the overall response to ruxolitinib in children with GVHD is good, only a small number of cases have been reported on the treatment efficacy in children. ${ }^{8,9}$

Therefore, we conducted this study to analyze and summarize the clinical efficacy of ruxolitinib in treating children with refractory GVHD that had responded poorly to steroid and second-line treatment. The efficacy of ruxolitinib on transplantation-associated thrombotic microangiopathy (TA-TMA) was also investigated.

\section{Patients and Methods}

\section{Patients}

The patients who underwent allo-HSCT in the Beijing Children's Hospital from November 2017 to Jun 2019 and later developed SR-GVHD were enrolled in this study. This study was conducted following the Declaration of Helsinki and approved by the Institutional Review Board of the Beijing Children's Hospital, Capital Medical University (2019-k-287). This study was conducted in accordance with the declaration of Helsinki. Written informed consent was obtained from all patient guardians.

\section{Inclusion and Exclusion Criteria}

Inclusion criteria: (1) Patients who underwent allo-HSCT and later developed SR-GVHD; and (2) Patients who have signed informed consent. Exclusion criteria: (1) Patients who had severe infections; (2) Patients who had severe liver or kidney dysfunctions; (3) Patients with incomplete data; and (4) Patients who had received other anti-GVHD treatment and experienced improvement within 2 weeks before the ruxolitinib treatment.

\section{Definitions and Criteria}

The clinical grade of GVHD was classified according to aGVHD and cGVHD criteria. $^{10,11}$ SR-aGVHD was defined as a progressive disease after 3 days of high-dose systemic glucocorticoid ( $2 \mathrm{mg} / \mathrm{kg} / \mathrm{d}$ of methylprednisolone) treatment, a stable disease after 7 days of treatment or partial response (PR) after 14 days of treatment. SRcGVHD was defined as a progressive disease after 2 weeks of prednisone $1 \mathrm{mg} / \mathrm{kg} /$ day and stable disease after 1-8 weeks of prednisone $0.5 \mathrm{mg} / \mathrm{kg} /$ day and the prednisone maintenance dose were greater than $0.5 \mathrm{mg} / \mathrm{kg} / \mathrm{day}{ }^{2,12}$

\section{The Ruxolitinib Dosage}

The drug dosing was based on the clinical experience of Khandelwal et $\mathrm{al}^{8}$ in treating GVHD with ruxolitinib in children: $2.5 \mathrm{mg}$ for those with a bodyweight of $<25 \mathrm{~kg}$ and $5 \mathrm{mg}$ for those with a bodyweight of $\geq 25 \mathrm{~kg}$. The drug was orally administered qd or bid. For worsening conditions, the maximum dose could be increased up to $10 \mathrm{mg}$ bid. At the beginning of the treatment and during the dose increments, however, the dose of ruxolitinib was reduced by half or maintained in patients with the following conditions: low blood cell counts, infection or viremia and/or those receiving an azole antifungal treatment. After the clinical symptoms were improved for at least 1 week, ruxolitinib was tapered or continued at the maintenance dosage. Taper schedule and the duration of maintenance based on the clinical manifestation and symptoms of GVHD and is up to the core group. In general, it will tapered $1 / 4-1 / 8$ of the previous dosage. The duration of ruxolitinib may be 3 months to 1 year.

\section{The Evaluation of the Treatment Response}

For patients with aGVHD, treatment failure referred to the discontinuation of ruxolitinib due to any side effects within 4 weeks after the commencement of treatment or if any organ symptoms of GVHD progressed. Complete response (CR) to ruxolitinib treatment required the resolution of all manifestations of aGVHD and PR was defined as the reduction of GVHD severity by at least one grade without additional therapy and worsening in other organ systems for at least 3 weeks.

For patients with cGVHD, CR was defined as the complete disappearance of GVHD symptoms and PR was defined as symptom relief (the score of organ improved at least 1) after 4 weeks of treatment and 
tapering of immunologic agents. ${ }^{4,12}$ Treatment failure was defined as the discontinuation of medication due to obvious side effects within 4 weeks of treatment or switching to other chemotherapy regimens because of disease progression or tumor recurrence after 4 weeks of treatment.

The treatment response was evaluated monthly after 4 weeks of treatment. The optimal response during the treatment period was recorded. The discontinuation of ruxolitinib before the completion of the 4-week therapy because of ruxolitinib toxicity, and not because of a lack of response, was considered treatment failure. Any case that was not categorized as CR, PR or treatment failure was considered to be no response (NR). The overall response rate (ORR) was presented as the percentage of patients who achieved CR or PR after administering the treatment among all patients.

\section{The Assessment of Adverse Events}

According to the literature, ${ }^{4,7}$ the common adverse events of ruxolitinib included cytopenia, allergic reaction, liver damage, and secondary viral/bacterial infections. We analyzed the adverse events that occurred during the ruxolitinib treatment according to the Common Terminology Criteria for Adverse Events, version 5.0 (http://ctep.can cer.gov).

\section{The Clinical Follow-Ups}

The follow-ups were carried out until Jun 1, 2019. The follow-up data were obtained from hospital records, outpatient visits, and online/telephone communication.

\section{Statistical Analysis}

We used the software program SPSS 22.0 (IBM Corp., Armonk, NY, USA) and GraphPad Prism, version 7.0 (GraphPad Software, San Diego, CA, USA) to conduct the statistical analysis. Continuous variables were expressed as mean \pm SD and discontinuous variables were expressed as a percentage (\%). Overall survival (OS) was defined as the time from the commencement of the ruxolitinib treatment until death from any cause or the last follow-up visit. Event-free survival (EFS) was defined as the time from the commencement of the ruxolitinib treatment until the occurrence of any of the following events: death from any cause, disease progression, the restarting of chemotherapy or the addition of other immunosuppressive agents due to clinical requirements, the recurrence of $\mathrm{GVHD}$, tumor recurrence or treatment failure, whichever came first, or the last follow-up. OS and EFS were calculated using the Kaplan-Meier method and were compared between the two groups using the Log rank test. The treatment response rate and incidence of adverse events were compared using the chi-squared test or Fisher's exact test. A $P$-value of $<0.05$ was considered statistically significant.

\section{Results}

\section{The General Characteristics}

A total of 53 pediatric patients were included in this study. Among these 53 patients, there were 34 boys and 19 girls. The patients' age ranged from 7 months to 18 -years-old. HLA-matched peripheral blood stem cell transplantation was performed in 8 patients and HLA-haploidentical bone marrow plus peripheral blood stem cell transplantation was performed in 45 patients. Seventeen patients developed grades II (4), III (12), IV (1) aGVHD and 36 patients experienced moderate (28) or severe (8) cGVHD (Table 1). GVHD mainly affected the skin, gastrointestinal tract, and liver. The lungs, mouth, and eyes were also affected in 7 (13.2\%) patients. No obvious abnormality was detected in the children's joints, serosa or genital organs. The median number of second-line agent received prior to ruxolitinib was 3 (range 2-4) in aGVHD group and 3 (rang 2-6) in cGVHD group. The median duration of ruxolitinib treatment was 283 days (range 15-500 days). And the median duration of follow-up time was 304 days (rang 93-508 days). In total, 12 children (cases 3, 6, 12, 14, 16, 23, 24, 31, 39, 43, 46, and 52) had lower blood cell counts, infections or required the use of azoles when they started the ruxolitinib therapy, and therefore, the starting dose in these patients was reduced by half. A total of 15 patients ( 5 in the aGVHD group and 10 in the cGVHD group) received ruxolitinib at the maximum dosage.

\section{The Patients' Response to Ruxolitinib Treatment}

In total, the ORR was $75.5 \%$ (40/53, Table 2$)$. The ORR was $64.7 \%$ (11/17) among the patients with aGVHD, including 6 PRs (35.3\%) and 5 CRs (29.4\%). All immunosuppressive agents were withdrawn in 1 patient (case 2) and the number and dose of immunosuppressive agents were reduced in the remaining 10 patients. CR was achieved in 10 out of $36(27.8 \%)$ patients with cGVHD and the immunosuppressive agents were gradually tapered in these patients. At the end of the follow-up, the immunosuppressive agents were completely withdrawn in 5 
Table I Characteristics of Patients with a/cGVHD

\begin{tabular}{|c|c|c|}
\hline Index & $\begin{array}{l}\text { aGVHD } \\
(n=17)\end{array}$ & $\begin{array}{l}\text { cGVHD } \\
(n=36)\end{array}$ \\
\hline Age, year, median (range) & $7(0.9-13.6)$ & $\begin{array}{l}6.9 \\
(3.3-17.5)\end{array}$ \\
\hline \multicolumn{3}{|l|}{ Gender } \\
\hline Male & 12 & 22 \\
\hline Female & 5 & 14 \\
\hline \multicolumn{3}{|l|}{ Disease } \\
\hline AML & 4 & 2 \\
\hline Relapsed ALL & 3 & 4 \\
\hline HLH & 4 & 4 \\
\hline Osteopetrosis & 2 & 5 \\
\hline Fanconi anemia & I & - \\
\hline MDS & I & - \\
\hline AA & 2 & 13 \\
\hline $\mathrm{NHL}$ & - & 5 \\
\hline CML & - & I \\
\hline$\beta$-thalassemia & - & 2 \\
\hline \multicolumn{3}{|l|}{ Relation } \\
\hline Related & 16 & 31 \\
\hline Unrelated & I & 5 \\
\hline \multicolumn{3}{|l|}{ Stem source } \\
\hline $\mathrm{BM}+\mathrm{PBSC}$ & 16 & 29 \\
\hline PBSC & I & 7 \\
\hline Maximum grade GVHD & $\begin{array}{l}\text { Grade II: } 4 \\
\text { Grade III: I2 } \\
\text { Grade IV: I }\end{array}$ & $\begin{array}{l}\text { Moderate: } 28 \\
\text { Severe: } 8\end{array}$ \\
\hline $\begin{array}{l}\text { Median number of regimens received } \\
\text { prior to Ruxolitinib (range) }\end{array}$ & $3(2-4)$ & $3(2-6)$ \\
\hline
\end{tabular}

Abbreviations: GVHD, graft-versus-host disease; aGVHD, acute GVHD; cGVHD, chronic GVHD; AML, acute myelogenous leukemia; ALL, acute lymphoblastic leukemia; $\mathrm{HLH}$, hemophagocytic lymphocytic histiocytosis; MDS, myelodysplastic syndrome; AA, aplastic anemia; NHL, non-Hodgkin lymphoma; CML, chronic myelogenous leukemia; BM, bone marrow; PBSC, peripheral blood stem cells.

patients (cases 24, 33, 35, 43 and 46) and 4 patients (cases $19,20,23$, and 48) received ruxolitinib treatment at the maintenance dose alone. A total of 14 patients $(39 \%)$ discontinued steroids and 8 patients $(22.2 \%)$ reduced steroids. PR was achieved in 19 patients (52.8\%), among which 3 patients (cases 41, 42, and 45) received the ruxolitinib treatment at the maintenance dose alone. The ORR was not significantly different between the aGVHD and cGVHD groups $(64.7 \%$ vs $80.6 \%$, Fisher's exact test, $P=0.306)$. The treatment response rates did not differ significantly among the patients with different primary diseases in both the aGVHD (Fisher's exact test, $P=1.000$ ) and cGVHD groups (Fisher's exact test, $P=0.609$ ). And
Table 2 Response to Ruxolitinib Treatment

\begin{tabular}{|l|l|l|l|}
\hline Ruxolitinib Indication & $\begin{array}{l}\text { aGVHD } \\
(\mathbf{n}=17)\end{array}$ & $\begin{array}{l}\text { cGVHD } \\
(\mathbf{n}=36)\end{array}$ & P value \\
\hline $\begin{array}{l}\text { Response } \\
\text { ORR }\end{array}$ & $64.7 \%$ & $80.6 \%$ & 0.306 \\
CR & 5 & 10 & 1.000 \\
PR & 6 & 19 & 0.257 \\
NR & 0 & 5 & 0.163 \\
Treatment Failure & 6 & 2 & 0.010 \\
\hline $\begin{array}{l}\text { Duration of Ruxolitinib } \\
\text { Treatment days, median } \\
\text { (range) }\end{array}$ & 87 & 207 & 0.407 \\
\hline Time to best response & $(14-305)$ & $(28-373)$ & \\
Treatment days, median & $28(9-56)$ & $68(37-156)$ & - \\
(range) & & & \\
\hline GVHD relapse & 4 & 9 & 1.000 \\
\hline Tumor relapse & 0 & 1 & 1.000 \\
\hline Current status & 13 & 36 & 0.008 \\
Alive & 4 & 0 & \\
\hline Dead & & & \\
\hline
\end{tabular}

Abbreviations: GVHD, graft-versus-host disease; aGVHD, acute GVHD; cGVHD, chronic GVHD; ORR, overall response rate; CR, complete response; PR, partial response; NR, no response.

the ideal response organ were skin, cutaneous, hepatic and gastro-intestinal in aGVHD grades II-III and moderate of cGVHD.

Although NR was not observed in the children with aGVHD, 6 patients experienced treatment failure; the ruxolitinib treatment was discontinued 2-4 weeks after the start of treatment because of significant decreases in blood cell counts (grade 4 pancytopenia). Five children with cGVHD were unresponsive to the ruxolitinib treatment and 2 patients experienced treatment failure (grade 4 pancytopenia or tumor relapse). Although there was no significant difference in the incidence of NR between the aGVHD and cGVHD groups ( 0 vs $13.9 \%$, Fisher's exact test, $P=0.163$ ), the incidence of treatment failure was significantly higher in the aGVHD group than in the cGVHD group (35.3\% vs 5.6\%, Fisher's exact test, $P=0.010)$. But in the aGVHD group, most of the failures were due to toxicity.

The recurrence of GVHD after the ruxolitinib treatment was observed in 13 (32.5\%) out of 40 patients with CR or PR (11 patients in the aGVHD group and 29 patients in the cGVHD group). In particular, recurrence occurred in 4 patients with aGVHD (4/11, 36\%), including 
3 children (cases 4, 8, and 10) with CRs and 1 patient with PR (case 16), whereas recurrence occurred in 9 patients with cGVHD (9/29, 31\%), including 1 child (case 20) with $\mathrm{CR}$ and 8 children (cases 26, 27, 28, 29, 32, 34, 36, and 37) with PRs. Thus, the recurrence rate of GVHD after ruxolitinib treatment was not significantly different between the two groups (Fisher's exact test, $P=1.000$ ).

\section{The Adverse Events During the Ruxolitinib Treatment}

In the aGVHD group, 16 patients (94.1\%) experienced drugrelated side events, including elevated alanine aminotransferase levels $(n=4)$ and cytopenia $(n=12)$. Eleven patients $(64.7 \%)$ also developed infections: Epstein-Barr Virus (EBV) viremia $(\mathrm{n}=2)$, cytomegalovirus $(\mathrm{CMV})$ viremia $(\mathrm{n}=6), \mathrm{BK}$ virus $(\mathrm{BKV})$ infection $(\mathrm{n}=2)$, and tuberculosis $(n=1)$. In the cGVHD group, 11 patients $(30.6 \%)$ experienced drug-related side effects including elevated alanine aminotransferase levels $(n=3)$ and cytopenia $(n=12)$. Five patients also developed other infections: bacterial infection $(\mathrm{n}=2), \mathrm{CMV}$ viremia $(\mathrm{n}=2)$, and Pneumocystis carinii pneumonia ( $\mathrm{n}=1$, Table 3 ). The incidence of adverse events was significantly higher in the aGVHD group than in the cGVHD group (Pearson's chi-squared test, $\chi^{2}=18.668 ; P=0.000015$ ).

\section{The Response to the Ruxolitinib Treatment in Children with TA-TMA}

In the 53 patients with GVHD, 11 patients (including 8 patients with aGVHD and 3 patients with cGVHD) developed TA-TMA, which was diagnosed according to the Bone and Marrow Transplant Clinical Trials Network criteria. ${ }^{13}$ After the diagnosis was confirmed, plasma

Table 3 Adverse Events During Ruxolitinib Treatment

\begin{tabular}{|l|l|l|}
\hline \multirow{2}{*}{ Variables } & aGVHD(n= I7) & cGVHD(n=36) \\
\cline { 2 - 3 } & $\%($ Number) & $\%($ Number) \\
\hline Cytopenia & $70(12)$ & $11(4)$ \\
Liver function injury & $29(5)$ & $8(3)$ \\
EBV reactivation & $12(2)$ & $/$ \\
CMV reactivation & $35(6)$ & $3(1)$ \\
BKV infection & $12(2)$ & $/$ \\
TB infection & $6(1)$ & $/$ \\
Bacterial infection & $/$ & $6(2)$ \\
PCP & $/$ & $3(I)$ \\
Malignancy relapse & $/$ & $3(I)$ \\
\hline
\end{tabular}

Abbreviations: GVHD, graft-versus-host disease; aGVHD, acute GVHD; cGVHD, chronic GVHD; EBV, Epstein-Barr virus; CMV, cytomegalovirus; BKV, BK virus; TB, tuberculosis; PCP, Pneumocystis carinii pneumonia. exchange and treatment with rituximab and defibrotide were applied according to the children's specific disease condition and the dose of the calcineurin inhibitor was reduced or the drug was withdrawn. Cyclosporine and tacrolimus were not used in the treatment of TA-TMA.

TMA was effectively controlled in all 11 children and the ORR for these patients was $90.9 \%$ (10/11, including 6 with $\mathrm{CR}$ and 4 with PR). One patient failed treatment because of severe cytopenia. Compared with the other patients with GVHD, the ORR and incidence of treatment failure were not significantly different in the TA-TMA group (Fisher's exact test, $P=0.257$ and $\mathrm{P}=0.674$, respectively). During the use of ruxolitinib, the children developed varying degrees of cytopenia $(n=3)$, impaired liver function $(n=4), C M V$ viremia $(n=3), E B V$ viremia $(n=2)$, and BKV infection ( $\mathrm{n}=2$, Table 4). No obvious difference compared with the other patients was identified.

\section{Tumor Recurrence}

During the ruxolitinib treatment, 1 patient (1.9\%; case 38 ) experienced a relapse of a primary tumor (ALL) and a new chemotherapy regimen was started. This patient is still alive.

\section{Survival Rates}

The median follow-up duration for the 53 patients was 43.43 weeks (range: 13.29-73.57 weeks). The median follow-up time of the 17 children in the aGVHD group was 40.57 weeks (range: 13.29-72.57 weeks). During this period, 4 patients with primary AML or relapsed ALL (three cases with treatment failure and 1 with PR) died because of severe complications of GVHD. In the 6th month of the ruxolitinib treatment, OS and EFS were $92.3 \% \pm 7.4 \%$ and $69.3 \% \pm 11.5 \%$, respectively.

The median follow-up duration was 44.36 weeks (range: 18-73.57 weeks) among the 36 children with cGVHD. The primary disease (ALL) recurred during treatment in 1 patient (case 38) who remained alive after being retreated with chemotherapy. No deaths occurred in the cGVHD group after the ruxolitinib treatment. The 6-month OS and EFS were $100 \%$ and $96.9 \% \pm$ 3.1\%, (Figure 1A and B). The median predicted EFS times were 44.43 (95\% CI: 35.6-53.25) and $53.43(95 \%$ CI: 47.82-59.04) weeks in the aGVHD and cGVHD groups, respectively.

\section{Discussion}

The outcomes of this study presented that the overall response rate (ORR) to ruxolitinib was $75.5 \%$. The ORR 
Table 4 Characteristics of Patients with Transplantation-Associated Thrombotic Microangiopathy

\begin{tabular}{|c|c|c|c|c|c|c|c|c|c|c|c|c|}
\hline $\mathbf{P t}$ & $\begin{array}{l}\text { ABO } \\
\text { Blood Type }\end{array}$ & $\begin{array}{l}\text { MNC } \\
\left(\times 10^{8} / \mathrm{kg}\right)\end{array}$ & $\begin{array}{l}\mathrm{CD} 34^{+}\left(\times 10^{6}\right) \\
\mathrm{kg})\end{array}$ & $\begin{array}{l}\text { Time to TA- } \\
\text { TMA(Days) }\end{array}$ & $\begin{array}{l}\text { Hb } \\
(\mathrm{g} / \mathrm{L})\end{array}$ & $\begin{array}{l}\text { PIt } \\
\left(\times 10^{9} / L\right)\end{array}$ & $\begin{array}{l}\text { LDH } \\
\text { (U/L) }\end{array}$ & $\begin{array}{l}\text { CsA } \\
\text { (ug/L) }\end{array}$ & $\begin{array}{l}\text { FK506 } \\
(\mathrm{ng} / \\
\mathrm{mL})\end{array}$ & $\begin{array}{l}\text { TMA } \\
\text { Organ }\end{array}$ & $\begin{array}{l}\text { Response } \\
\text { to } \\
\text { Ruxolinitib }\end{array}$ & $\begin{array}{l}\text { Adverse } \\
\text { Effects }\end{array}$ \\
\hline 1 & Incompatible & 9.39 & 8.01 & 14 & 75 & 50 & 671 & I & 26.8 & Renal & PR & CMV/Liver \\
\hline 2 & Incompatible & 17.49 & 8.0 & 61 & 61 & 5 & 3861 & 365.5 & 1 & Liver+Renal & $C R$ & $\begin{array}{l}\text { CMV/BKV/ } \\
\text { Liver/cytopenia }\end{array}$ \\
\hline 3 & Incompatible & 3.11 & 10.07 & 19 & 70 & 29 & 1766 & 517.2 & 1 & Liver+Lung & $C R$ & EBV/Liver \\
\hline 4 & Compatible & 7.07 & 11.79 & 11 & 59 & 10 & 861 & 324.7 & 1 & Liver+Renal & CR & Cytopenia \\
\hline 5 & Compatible & 4.49 & 7.5 & 43 & 67 & 14 & 905 & 451 & 1 & NS+Liver & Failure & BKV/cytopenia \\
\hline 7 & Incompatible & 7.51 & 6.07 & 40 & 89 & 30 & 677 & 108.8 & 1 & Renal & PR & $\mathrm{CMV/cytopenia}$ \\
\hline 8 & Incompatible & 11.27 & 4.12 & 18 & 90 & 21 & 770 & 436.5 & 1 & Liver & $C R$ & EBV \\
\hline 11 & Incompatible & 10.22 & 3.52 & 63 & 73 & 36 & 629 & 144.7 & 1 & NS+Liver & PR & - \\
\hline 18 & Compatible & 10.51 & 5.09 & 257 & 77 & 29 & 937 & 86.6 & 1 & Renal & PR & - \\
\hline 35 & Incompatible & 32.0 & 10.25 & 205 & 58 & 10 & 1652 & I & 13.5 & Renal & $C R$ & Liver \\
\hline 48 & Compatible & 11.22 & 7.26 & 156 & 101 & 95 & 552 & 64.5 & 1 & Liver & $C R$ & - \\
\hline
\end{tabular}

Abbreviations: BKV, BK virus; CR, complete response; CsA, cyclosporin A; CMV, cytomegalovirus; EBV, Epstein-Barr virus; FK506, tacrolimus; LDH, lactate dehydrogenase; MNC, mononuclear cell; NS, nervous system; PR, partial response; TA-TMA, transplantation-associated thrombotic microangiopathy.

was $64.7 \%$ in the aGVHD group and $80.6 \%$ in the cGVHD group. A significantly higher incidence of treatment failure was observed in the aGVHD group. The incidence of adverse events was higher in the aGVHD group than that in the cGVHD group. Meanwhile, the prognosis of children with cGVHD was superior to that of children with aGVHD after the treatment with ruxolitinib. During the ruxolitinib treatment, only $1.9 \%$ of patients suffered a relapse of the primary tumor. Following the ruxolitinib treatment without cyclosporine, tacrolimus or other anti-GVHD treatments, TMA was effectively controlled and the ORR reached $90.9 \%$.

After allo-HSCT, a/cGVHD seriously affects treatment efficiency and patient survival. Glucocorticoids remain the first-line treatment for GVHD but the response rate is only approximately $50 \%$. For SR-GVHD, the drugs used in second-line treatment mainly include ATG, MMF,
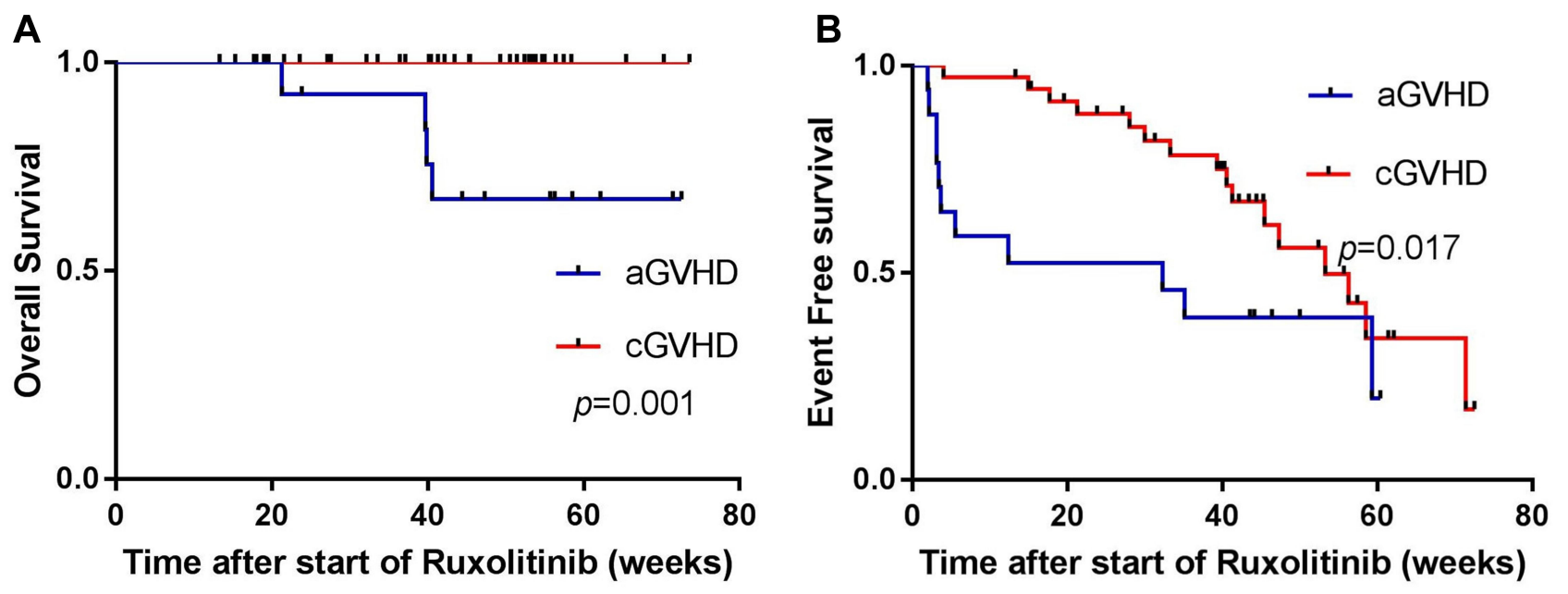

Figure I The survival of patients with graft-versus-host disease (GVHD) treated with ruxolitinib: (A) overall survival, (B) event-free survival. Abbreviations: aGVHD, acute GVHD; cGVHD, chronic GVHD. 
methotrexate, tacrolimus, anti-CD25 antibody (daclizu$\mathrm{mab}$ ), and rapamycin. However, the response rate to these treatments is only approximately $30 \%{ }^{4,14,15}$ Currently, there is no widely recognized second-line treatment. The JAK/signal transducers and activators of transcription (JAK/STAT) pathway, a downstream signaling pathway for multiple inflammatory factors, is involved in mediating the biological effects of multiple cytokines. ${ }^{16}$ In vivo, pharmacologic inhibition of IFN $\gamma \mathrm{R}$ signaling with inhibitors of JAK1/JAK2, which are mediators of IFN $\gamma \mathrm{R}$ signaling, results in the decreased expression of CXCR3 and reduced GVHD and improved survival after alloHSCT. $^{6}$ In mice, blocking JAK $1 / 2$ signaling reduces the proliferation of effector $\mathrm{T}$ cells, inhibits pro-inflammatory cytokines that respond to alloantigens, and increases the number of FoxP ${ }^{+}$Treg cells to induce immune tolerance while ultimately increasing the survival of aGVHD mice and decreasing both the pathological grade of GVHD and plasma pro-inflammatory cytokine levels. ${ }^{3}$ Therefore, treatments targeting the JAK/STAT pathway may alleviate or even completely control GVHD.

Ruxolitinib is an oral JAK $1 / 2$ inhibitor and in addition to its anti-GVHD effect, ruxolitinib can also preserve graftversus-leukemia/graft-versus-tumor (GVL/GVT) function. ${ }^{17,18}$ Choi et $\mathrm{al}^{17}$ reported that ruxolitinib reduces GVHD and preserves the beneficial GVL effect in two different murine MHC-mismatched allo-HSCT models and using two different murine leukemia models (lymphoid leukemia and myeloid leukemia). Carniti et al ${ }^{18}$ also examined the effect of JAK1/JAK2 modulation by ruxolitinib in a mouse model of fully MHC mismatched bone marrow transplant comprising in vivo tumor inoculation; and found that ruxolitinib improved both overall survival and aGVHD pathologic score at target organs of treated mice. In addition, treatment with ruxolitinib was associated with a preserved GVT effect, as evidenced by reduction of tumor burden and increase of survival time. In a previous study, the outcome of 95 patients who received oral ruxolitinib at 19 stem cell transplant centers were analyzed, ${ }^{4}$ including 54 patients with SR-aGVHD (grades III-IV) and 41 patients with SR-cGVHD (moderate or severe). The findings of this study showed that the ORR was $81.5 \%(44 / 54)$ and the CR rate was $46.3 \%(25 / 54)$ in patients with aGVHD; the ORR reached $85.4 \%(35 / 41)$ in patients with cGVHD, and the CR rate was 7.3\% (3/41). Meanwhile, the levels of inflammation-related markers also decreased. Khoury et $\mathrm{al}^{19}$ reported a more effective result, total of 19 allograft recipients with moderate/severe steroid- dependent chronic GVHD received ruxolitinib, 18 achieved $\mathrm{PR}$ and $1 \mathrm{CR}$. And more importantly reduction to physiologic doses/discontinuation of prednisone was possible in $\sim 90 \%$ of patients. However, no pediatric patients were included in this study. Khandelwal et $\mathrm{al}^{8}$ retrospectively analyzed the effectiveness of ruxolitinib in treating children with refractory aGVHD after allo-HSCT. The ORR was $45 \%$ in 13 children, including 1 patient achieving CR and four achieving PR. González-Vicent et $\mathrm{al}^{9}$ reported 13 cases of aGVHD and 9 cases of cGVHD with ORR of $77 \%$ and $89 \%$, respectively. Shi et $\mathrm{al}^{20}$ retrospectively analyzed 12 pediatric patients with SR-GVHD after allo-HSCT (including 1 patient treated with ruxolitinib at both the aGVHD and cGVHD stages). The ORR was $80 \%$ in the 5 patients with aGVHD and $87.5 \%$ in the 8 patients with cGVHD.

Several clinical trials for SR-a/cGVHD with ruxolitinib treatment are currently underway in many countries. ${ }^{21}$ These studies are based on Registration, Evaluation, Authorization and Restriction of Chemicals (REACH) compliance, phases $1 / 2 / 3$. The encouraging results from $\mathrm{REACH} 1$ indicated that ruxolitinib is the preferred treatment for SR-aGVHD with an best ORR of $73.2 \%{ }^{22}$ Zeiser et $\mathrm{al}^{23}$ reported that the REACH2 (a multicenter, randomized, open-label, Phase 3 trial) results recently, the overall response of SR-aGVHD at day 28 was significantly higher in the ruxolitinib group than in the control group (62\% [96 of 154 patients] vs $39 \%$ [61 of 155 patients]; and the percentage of CR was 34\% vs $19 \%$. However, the age of the patients enrolled in REACH1 was $\geq 12$ years. Therefore, for younger children, prospective studies with large sample sizes are needed to further confirm the efficacy and toxicity of ruxolitinib.

In the present study, we retrospectively collected the clinical data of 53 children with GVHD treated with ruxolitinib. The overall ORR was $75.5 \%$, which was similar to the findings of studies conducted by Zeiser et al in adults $(81.5 \%)^{4}$ and González-Vicent in children $(77 \%$ and $89 \%$ in patients with aGVHD and cGVHD, respectively) ${ }^{9}$ and slightly higher than that reported by Khandelwal et al in children $(45 \%){ }^{8}$ As demonstrated in the present study, there was no significant difference in ORR between the aGVHD and cGVHD groups after ruxolitinib treatment; however, the cGVHD group had significantly higher 6-month OS and EFS rates than the aGVHD group, suggesting that ruxolitinib could more efficiently control cGVHD, which is in line with previous reports. ${ }^{4,5}$ However, the specific mechanism is unknown. It may be explained by the better prognosis of patients with cGVHD and/or by the different organs/systems affected in these two conditions. 
The main target organs of cGVHD in the present study were the skin, liver, and eyes; whereas, the intestinal rejection was more common in patients with aGVHD. Severe diarrhea and severe intestinal GVHD may lead to poor absorption and utilization of oral ruxolitinib. Neumann et $\mathrm{al}^{24}$ and Khandelwal et $\mathrm{al}^{8}$ also reported that patients with intestinal GVHD responded poorly to ruxolitinib treatment. During the treatment of cGVHD, ruxolitinib can reduce the complications associated with long-term steroid use. In the present study, ruxolitinib was still effective in 2 patients with cGVHD (cases 41 and 42) 5 years after transplantation.

Ruxolitinib is mainly used in adults and the optimal dosage in children remains unclear. In a previous clinical study, ${ }^{25}$ children with refractory hematologic malignancies could tolerate oral ruxolitinib of $15-50 \mathrm{mg} / \mathrm{m}^{2}$ bid. The dose of ruxolitinib for bone marrow fibrosis in adults also reached $30-50 \mathrm{mg} /$ day. $^{26}$ However, the ruxolitinib dose for GVHD in adults is only $10-20 \mathrm{mg} /$ day. Based on this dosage, the calculated maximum dose in children will exceed the dose for adults with GVHD. Therefore, we referred to the pediatric dose of ruxolitinib for GVHD proposed by Khandelwal et al. ${ }^{8}$ Meanwhile, we intended to use ruxolitinib more positively according to the results of previous studies. For instance, ruxolitinib treatment was administered to any patient with refractory GVHD, yet few studies have described the pharmacokinetics of ruxolitinib. González-Vicent ${ }^{9}$ found that the absorption and bioavailability of ruxolitinib were good in children. However, detailed studies are needed to interpret the characteristics of this drug's pharmacokinetics.

TA-TMA is a fatal complication after allo-HSCT. Its clinical manifestations include thrombocytopenia, microvascular hemolytic anemia, microvascular thrombosis, and multiple organ dysfunction. The reported incidence of TMA after allo-HSCT varies from 0.5 to $76 \% .^{27,28}$ The specific mechanism of TA-TMA remains unknown. It is currently believed that its risk factors include a myeloablative conditioning regimen, allo-HSCT (especially haplo-HSCT), the use of calcineurin inhibitors, GVHD, and infection. ${ }^{27,29}$ Most cases of TA-TMA are complicated by GVHD and TMA and GVHD have many similarities in their pathogenic mechanisms and clinical manifestations. Because anemia, thrombocytopenia, renal impairment, and changes in mental status are common and may have multiple causes in the transplant population, diagnosis and therapy may be difficult. Thus, the selection of alternative drugs is particularly important. A poor therapeutic effect on TMA can lead to recurrent or aggravated GVHD. Because the pathogenesis of TATMA is complex and not well elucidated, there is currently no optimal treatment for the disease. The main treatments include symptomatic and etiological therapies. Zhao et $\mathrm{al}^{30}$ reported that some allo-SCT recipients cannot tolerate calcineurin inhibitors, then they change into ruxolitinib, not only appear particularly promising as CNI-free GVHD prophylaxis, but also for alleviating microvascular endothelial injury by inhibiting the release of inflammatory cytokines. For patients with complicated TA-TMA in the present study, ruxolitinib was administered after the calcineurin inhibitors were withdrawn. As a result of this, both TMA and GVHD symptoms were effectively controlled. Although the reappearance of GVHD symptoms was noted in some children, no TMA recurrence was observed. Thus, our findings offered a new immunotherapy option for TA-TMA complicated with GVHD.

Ruxolitinib has some serious side effects when used in a clinical setting. Zeiser et $\mathrm{al}^{4}$ reported that ruxolitinib caused cytopenia as well as CMV reactivation (55.6\% and 33.3\%, respectively, in patients with aGVHD and $17.1 \%$ and $14.6 \%$, respectively, in patients with cGVHD). Khandelwal et $\mathrm{al}^{8}$ also identified the main side effects of ruxolitinib in children as liver impairment, moderate-to-severe cytopenia, and a variety of infection-related complications. However, these adverse effects are not specific to ruxolitinib; in fact, the incidence of such adverse events can reach up to $73 \%$ during the use of other immunosuppressive agents (especially MMF). ${ }^{31}$ During a previous study that evaluated the use of ruxolitinib in aGVHD patients, 16 pediatric patients $(94.1 \%)$ experienced adverse events (especially cytopenia, 70\%). Therefore, ruxolitinib may be added as early as possible in patients with aGVHD before the onset of thrombocytopenia to achieve better response rates and avoid serious adverse events. Cytopenia is a reversible condition that can be alleviated via symptomatic blood transfusion (and the eventual withdrawal of the drug). It is recommended that routine blood cell counts be performed. ${ }^{31}$ CMV viremia was another common side effect in the present study with an incidence of $35 \%$ which was similar to the findings reported by Zeiser et al. Therefore, CMV DNA copy levels should be closely monitored. Other adverse events included liver impairment (29\%), EBV viremia $(12 \%)$, and BKV infection (12\%). Eleven patients with cGVHD (30.5\%) experienced adverse events including cytopenia (11\%), liver impairment (8\%), CMV viremia (3\%), and various infections (Table 3). After the antiviral therapy and low-dose steroid therapy were administered to the patients, 
ruxolitinib remained effective and the incidence of adverse events was within the controllable range. It seemed that patients with aGVHD generally more likely to develop cytopenia than those with cGVHD. ${ }^{32}$ In addition to these common adverse events, Watson et a ${ }^{33}$ described the occurrence of fatal hypertriglyceridemia (triglycerides $>4000 \mathrm{mg} / \mathrm{dl}$ ) in an adult with cGVHD who was treated with both ruxolitinib and sirolimus. Such a combination is rarely used in our clinical practice and no significant increase in blood lipid levels has been observed after ruxolitinib treatment.

The application of novel immunosuppressive agents may attenuate the GVL/GVT effect, which may eventually lead to an increased risk of tumor recurrence. It has been reported that the incidences of malignancies in patients treated with ruxolitinib were $9.3 \%$ and $2.4 \%$ in patients with aGVHD and cGVHD, respectively, ${ }^{4,34}$ which is similar to those found after the use of other immunosuppressive agents. In the present study, there were 20 patients with primary malignant tumors, including 4 patients undergoing transplantation after experiencing tumor recurrence. During the ruxolitinib treatment, only 1 patient (1.8\%) experienced primary tumor (ALL) recurrence, which was less frequent than that observed in previous studies. Carniti et $\mathrm{al}^{18}$ found that ruxolitinib had a certain GVT effect while attenuating GVHD in mice, which may be related to the drug-induced apoptosis of A20 cells. Therefore, future studies should emphasize the GVL/GVT effects of ruxolitinib to prevent tumor recurrence or the occurrence of a second tumor. What's more, another Janus kinase (JAK)1-selective inhibitor - itacitinib was reported as well tolerated and demonstrated preliminary efficacy in patients with aGVHD in a PROSPECTIVE study, the OR were observed in $75 \%$ and $71 \%$ of patients with treatment-naive and treatment-refractory aGVHD, respectively, at day $28 .{ }^{35}$ In the future, more therapy will benefit patients.

\section{Limitations}

There were several limitations to this study. Firstly, this trial was only an retrospective trial and not a randomized controlled trial. Secondly, this study was only a single-center trial and the sample size was limited. Studies with large sample sizes and long-term follow-ups are necessary to further explore the clinical application of ruxolitinib in children. Thirdly, it is necessary to further explore strategies to strengthen the GVL/ GVT effect of ruxolitinib and reduce the risk of malignant tumor recurrence when administering ruxolitinib for GVHD. Fourthly, the clinical follow-up was short, and it is necessary to observe the clinical long-term prognosis.

\section{Conclusion}

Ruxolitinib may play a key role in treating refractory GVHD, especially cGVHD in children following HSCT treatment. The treatment can reduce the need to use steroids and other immunosuppressive agents for controllable adverse events. Ruxolitinib may also be used as a salvage treatment after GVHD or as an alternative treatment for TA-TMA.

\section{Acknowledgments}

The authors acknowledge all patients, families, and clinical staff at the Hematology Oncology Center of the Beijing Children's Hospital. We thank Andrea Baird, MD, from Liwen Bianji, Edanz Editing China for editing the English text draft of this manuscript.

\section{Funding}

This study was funded by the National Science and Technology Major Project for "Major New Drugs Innovation and Development" (2017ZX09304029). The funding body had no role in the design of the study and collection, analysis, and interpretation of data and in writing the manuscript.

\section{Disclosure}

The authors declare that they have no competing interests.

\section{References}

1. Martin PJ, Inamoto Y, Flowers ME, Carpenter PA. Secondary treatment of acute graft-versus-host disease: a critical review. Biol Blood Marrow Transplant. 2012;18(7):982-988. doi:10.1016/j.bbmt.2012.04.006

2. Bacigalupo A. Management of acute graft-versus-host disease. $\mathrm{Br}$ J Haematol. 2007;137(2):87-98. doi:10.1111/j.1365-2141.2007.06533.x

3. Spoerl S, Mathew NR, Bscheider M, et al. Activity of therapeutic JAK $1 / 2$ blockade in graft-versus-host disease. Blood. 2014;123 (24):3832-3842. doi:10.1182/blood-2013-12-543736

4. Zeiser R, Burchert A, Lengerke $\mathrm{C}$, et al. Ruxolitinib in corticosteroid-refractory graft-versus-host disease after allogeneic stem cell transplantation: a multicenter survey. Leukemia. 2015;29 (10):2062-2068. doi:10.1038/leu.2015.212

5. Ferreira AM, Pontes DSC, Pereira AD, et al. Ruxolitinib in steroid-refractory chronic graft-versus-host disease: experience of a single center. Bone Marrow Transplant. 2018;53(4):503-506. doi:10.1038/s41409-017-0068-2

6. Choi J, Ziga ED, Ritchey J, et al. IFN $\gamma \mathrm{R}$ signaling mediates alloreactive T-cell trafficking and GVHD. Blood. 2012;120(19):4093-4103. doi:10.1182/blood-2012-01-403196

7. Przepiorka D, Luo L, Subramaniam S, et al. FDA approval summary: ruxolitinib for treatment of steroid-refractory acute graft-versus-host disease. Oncologist. 2020;25(2):e328-e334. doi:10.1634/theoncologist.2019-0627

8. Khandelwal P, Teusink-Cross A, Davies SM, et al. Ruxolitinib as salvage therapy in steroid-refractory acute graft-versus-host disease in pediatric hematopoietic stem cell transplant patients. Biol Blood Marrow Transplant. 2017;23(7):1122-1127. doi:10.1016/j.bbmt.2017.03.029 
9. González Vicent M, Molina B, González de Pablo J, Castillo A, Díaz MÁ. Ruxolitinib treatment for steroid refractory acute and chronic graft vs host disease in children: clinical and immunological results. Am J Hematol. 2019;94(3):319-326. doi:10.1002/ajh.25376

10. Przepiorka D, Weisdorf D, Martin P, et al. 1994 Consensus conference on acute GVHD grading. Bone Marrow Transplant. 1995;15(6):825-828.

11. Jagasia MH, Greinix HD, Arora M, et al. National institutes of health consensus development project on criteria for clinical trials in chronic graft-versus-host disease: i. the 2014 diagnosis and staging working group report. Biol Blood Marrow Transplant. 2015;21(3):389-401. doi:10.1016/j.bbmt.2014.12.001

12. Lee SJ, Wolff D, Kitko C, et al. Measuring therapeutic response in chronic graft-versus-host disease. national institutes of health consensus development project on criteria for clinical trials in chronic graft-versus-host disease: IV. The 2014 response criteria working group report. Biol Blood Marrow Transplant. 2015;21(6):984-999. doi:10.1016/j.bbmt.2015.02.025

13. Ho VT, Cutler C, Carter S, et al. Blood and marrow transplant clinical trials network toxicity committee consensus summary: thrombotic micmangiopathy after hematopoietic stem cell transplantation. Biol Blood Marrow Transplant. 2005;11 (8):571-575. doi:10.1016/j.bbmt.2005.06.001

14. Hoda D, Pidala J, Salgado-Vila N, et al. Sirolimus for treatment of steroid-refractory acute graft-versus-host disease. Bone Marrow Transplant. 2010;45(8):1347-1351. doi:10.1038/bmt.2009.343

15. Furlong T, Martin P, Flowers ME, et al. Therapy with mycophenolate mofetil for refractory acute and chronic GVHD. Bone Marrow Transplant. 2009;44(11):739-748. doi:10.1038/bmt.2009.76

16. Rudolph J, Heine A, Quast T, et al. The JAK inhibitor ruxolitinib impairs dendritic cell migration via off-target inhibition of ROCK. Leukemia. 2016;30(10):2119-2123. doi:10.1038/leu.2016.155

17. Choi J, Cooper ML, Alahmari B, et al. Pharmacologic blockade of JAK1/ JAK2 reduces GvHD and preserves the graft-versus-leukemia effect. PLoS One. 2014;9(10):e109799. doi:10.1371/journal.pone.0109799

18. Carniti C, Gimondi S, Vendramin A, et al. Pharmacologic inhibition of JAK1/JAK2 signaling reduces experimental murine acute GVHD while preserving GVT effects. Clin Cancer Res. 2015;21 (16):3740-3749. doi:10.1158/1078-0432.CCR-14-2758

19. Khoury HJ, Langston AA, Kota VK, et al. Ruxolitinib: a steroid sparing agent in chronic graft-versus-host disease. Bone Marrow Transplant. 2018;53(7):826-831. doi:10.1038/s41409-017-0081-5

20. Shi X, Yan Y. Clinical observation on effect of Ruxolitinib as salvage therapy on steroid-refractory graft-versus-host disease in pediatric hematopoietic stem cell transplant patients. J China Pediatr Blood Cancer. 2018;23(4):211-224.

21. Jagasia M, Zeiser R, Arbushites M, Delaite P, Gadbaw B, Bubnoff NV. Ruxolitinib for the treatment of patients with steroid-refractory GVHD: an introduction to the REACH trials. Immunotherapy. 2018;10(5):391-402. doi:10.2217/imt-2017-0156

22. Jagasia M, Perales MA, Schroeder MA, et al. Results from REACH1, a single-arm Phase 2 study of ruxolitinib in combination with corticosteroids for the treatment of steroid-refractory acute graft-vs-host disease. Blood. 2018;132(Suppl 1):601. doi:10.1182/blood-2018-99116342

Drug Design, Development and Therapy

\section{Publish your work in this journal}

Drug Design, Development and Therapy is an international, peerreviewed open-access journal that spans the spectrum of drug design and development through to clinical applications. Clinical outcomes, patient safety, and programs for the development and effective, safe, and sustained use of medicines are a feature of the journal, which has also
23. Zeiser R, Bubnoff N, Butler J, et al. Ruxolitinib for glucocorticoid-refractory acute graft-versus-host disease. $N$ Engl $J$ Med. 2020;382(19):1800-1810. doi:10.1056/NEJMoa1917635

24. Neumann T, Schneidewind L, Weigel M, et al. Ruxolitinib for therapy of graft-versus-host disease. Biomed Res Int. 2019;2019:8163780. doi:10.1155/2019/8163780

25. Loh ML, Tasian SK, Rabin KR, et al. A Phase 1 dosing study of ruxolitinib in children with relapsed or refractory solid tumors, leukemias, or myeloproliferative neoplasms: a Children's Oncology Group phase 1 consortium study (ADVL1011). Pediatr Blood Cancer. 2015;62(10):1717-1724. doi:10.1002/pbc.25575

26. Jin J, Du X, Zhou DB, et al. Efficacy and safety of JAK inhibitor ruxolitinib in Chinese patients with myelofibrosis: results of a 1-year follow-up of A2202. Zhonghua Xиe Ye Xue Za Zhi. 2016;37 (10):858-863. doi:10.3760/cma.j.issn.0253-2727.2016.10.007

27. Stavrou E, Lazarus HM. Thrombotic microangiopathy in haematopoietic cell transplantation: an update. Mediterr J Hematol Infect Dis. 2010;2(3):e2010033. doi:10.4084/mjhid.2010.033

28. Laskin BL, Goebel J, Davies SM, et al. Small vessels, big trouble in the kidneys and beyond: hematopoietic stem cell transplantation-associated thrombotic microangiopathy. Blood. 2011;118(6):1452-1462. doi:10.1182/blood-2011-02-321315

29. Rosenthal J. Hematopoietic cell transplantation-associated thrombotic microangiopathy: a review of pathophysiology, diagnosis, and treatment. J Blood Med. 2016;7:181-186. doi:10.2147/JBM.S102235

30. Zhao Y, Shi J, Luo Y, et al. Calcineurin inhibitors replacement by ruxolitinib as graft-versus-host disease prophylaxis for patients after allogeneic stem cell transplantation. Biol Blood Marrow Transplant. 2020;26(5):e128-e133. doi:10.1016/j.bbmt.2020.01.012

31. Onishi C, Ohashi K, Sawada T, et al. A high risk of lifethreatening infectious complications in mycophenolate mofetil treatment for acute or chronic graft-versus-host disease. Int J Hematol. 2010;91 (3):464-470. doi:10.1007/s12185-010-0516-x

32. Abedin S, McKenna E, Chhabra S, et al. Efficacy, toxicity, and infectious complications in ruxolitinib-treated patients with corticosteroid-refractory graft-versus-host disease after hematopoietic cell transplantation. Biol Blood Marrow Transplant. 2019;S10838791(19) 30226-5.

33. Watson AP, Brunstein CG, Holtan SG. Life-threatening hypertriglyceridemia in a patient on ruxolitinib and sirolimus for chronic graft-versushost disease. Case Rep Transplant. 2018;2018:4539757. doi:10.1155/ 2018/4539757

34. Satake A, Schmidt AM, Nomura S. Inhibition of calcineurin abrogates while inhibition of mTOR promotes regulatory $\mathrm{T}$ cell expansion and graft-versus-host disease protection by IL-2 in allogeneic bone marrow transplantation. PLoS One. 2014;9(3):e92888. doi:10.1371/ journal.pone. 0092888

35. Schroeder MA, Khoury HJ, Jagasia M, et al. A phase 1 trial of itacitinib, a selective JAK1 inhibitor, in patients with acute graft-versus-host disease. Blood Adv. 2020;4(8):1656-1669. doi:10.1182/bloodadvances.2019001043 been accepted for indexing on PubMed Central. The manuscript management system is completely online and includes a very quick and fair peer-review system, which is all easy to use. Visit http://www. dovepress.com/testimonials.php to read real quotes from published authors. 